\title{
Collapse and revival of entanglement of two-qubit in superconducting quantum dot lattice with magnetic flux and inhomogeneous gate voltage
}

\author{
Sujit Sarkar \\ PoornaPrajna Institute of Scientific Research, \\ 4 Sadashivanagar, Bangalore 5600 80, India.
}

(Dated: December 7, 2018)

\begin{abstract}
We study the entanglement of a two-qubit system in a superconducting quantum dot (SQD) lattice in the presence of magnetic flux and gate voltage inhomogeneity. We observe a universal feature for the half-integer magnetic flux quantum which completely washes out the entanglement of the system both at zero and finite temperature. We observe that the ground state is always in a maximally entangled Bell state when there is no inhomogeneity in gate voltage in the superconducting quantum dot lattice. We find an important constraint in magnetic flux for ground state entanglement. We also observe few behavior of entanglement at finite temperature is in contrast with the zero temperature behavior.

PACS numbers: 03.65.. Ud, Entanglement and quantum nonlocality.

PACS numbers: 74.78. Na, Mesoscopic and nanoscale.

PACS numbers: 74.81. Fa, Superconducting wire networks.
\end{abstract}

\section{INTRODUCTION}

Recent years have witnessed a surge in research activity involving a close interaction between the subjects of quantum information science and many body condensed matter physics [1-4]. The key concept in quantum information is that of entanglement, a truly unique feature of quantum mechanical systems. Entanglement implies non-local correlations between quantum particles which do not have a classical counterpart. Entanglement measures provide an additional characterization of the many-body state in condensed matter. Let us consider the state of an interacting spin system as an example. Since the ground state wave function changes qualitatively in a quantum phase transition, it is of significant interest to probe how the genuine quantum aspects of the wave function, namely entanglement, changes as the transition point is traversed. The question that arises is whether the entanglement of the quantum system extends over the macroscopic distances as ordinary correlation do. In recent years, quantum phase transitions have been extensively studied in spin systems using well known quantum information theoretic measures like entanglement, fidelity, reduced fidelity, fidelity susceptibility. The fidelity typically drops in an abrupt manner at a quantum critical point indicating a dramatic change in the nature of the ground state wave function $[5,10]$.

We have been motivated by the extensive studies of entanglement physics and interesting results in spin system 11 24]. We decided to apply this concept of study in the different disciplines of quantum condensed matter quantum many-body system. Here we study the entanglement physics of two qubit superconducting quantum dot lattice. Before we proceed further, we would like to discuss the basic aspects of a superconduct- ing quantum dot lattice: Superconducting quantum dot lattice consists of array of superconducting grains. The superconducting grains are of nanoscale size, and different states of the superconducting grain are controlled by the ratio between charging energy and Josephson energy, while the average charge of the dot is controlled by the gate voltage. These are similar to the metallic and semiconductor quantum dot, where the different phases of the system depends on the ratio of Coulomb charging energy to the kinetic energy. Therefore the system can be described as a superconducting quantum dot (SQD) and the lattice as a superconducting quantum dot 25-31] lattice.

One can consider the Cooper pair of SQD as a charged boson. The Physics of SQD can therefore be described in terms of interacting bosons. Bosonic physics is more interesting and hard to understand than the fermionic physics. One can understand the starting point of fermionic physics from the standpoint of independent electron approximation, at least in higher dimensions, whereas to understand the interacting bosonic system, one has to introduce the interaction from the very beginning. At the same time quantum phase diagram of this SQD lattice is very rich with different quantum phases. Therefore the study of the entanglement physics for the SQD lattice for both zero and finite temperature is interesting in its own right [25-27].

It is well known from our previous studies that mesoscopic SQUID array can also be treated as the superconducting quantum dot lattice with modulated Josephson junction [25-26]. The authors of Ref. [28] have found the magnetic flux induced superconducting Coulomb blocked in mesoscopic SQUID array and also the magnetic flux induced superconductor- insulator quantum phase transition. Experimentally and also theoretically it reveals that the applied magnetic flux has 
an important effect in the SQD lattice system. We will see in due course of our study that the inhomoginity of the gate voltage plays an important role in entanglement to disentanglement (product state) transition. Therefore we are motivated to study entanglement physics of SQD lattice in the presence of applied magnetic flux and inhomoginity of gate voltage.

The plan of the manuscript is as follows. We present the model Hamiltonian and entanglement physics in section (II) of this manuscript. We present summary and conclusion in the section (III) of the manuscript.

\section{MODEL HAMILTONIAN FOR INHOMOGENEOUS SUPERCONDUCTING QUANTUM DOT LATTICE AND THE STUDY OF ENTANGLEMENT PHYSICS}

\section{A. Model Hamiltonian and Ground State} Analysis

At first we write down the model Hamiltonian of SQD lattice system with Josephson couplings having on-site charging energies and inter-site interactions in the presence of gate voltage and external magnetic flux. We also consider the inhomogeneity in the applied gate voltage. The Hamiltonian is written as

$$
H=H_{J 1}+H_{E C 0}+H_{E C 1} .
$$

We recast different parts of the Hamiltonian in quantum phase model as

$$
H_{J 1}=-E_{J 1}\left|\cos \left(\pi \frac{\Phi}{\Phi_{0}}\right)\right| \sum_{i} \cos \left(\phi_{i+1}-\phi_{i}\right),
$$

where $\phi_{i}$ and $\phi_{i+1}$ are quantal phase of the SQD at the point $\mathrm{i}$ and $\mathrm{i}+1$ respectively. as

$$
H_{E C 0}=\frac{E_{C 0}}{2} \sum_{i}\left(-i \frac{\partial}{\partial \phi_{i}}-\frac{N_{i}}{2}\right)^{2},
$$

where $E_{C 0}$ is the on-site charging energy, Now

$$
H_{E C 1}=E_{Z 1} \sum_{i} n_{i} n_{i+1},
$$

where $E_{Z 1}$ is the NN charging energies between the dots respectively. In the phase representation, $\left(-i \frac{\partial}{\partial \phi_{i}}\right)$ is the operator representing the number of Cooper pairs at the ith dot, and thus it takes only the integer values $\left(n_{i}\right)$. Here, Hamiltonian $H_{E C 0}$ accounts for the influence of gate voltage $\left(e N \sim V_{g}\right)$, where $e N$ is the average dot charge induced by the gate voltage. When the ratio $\frac{E_{J 1}}{E_{C 0}} \rightarrow 0$, the SQD array is in the insulating state having a gap of the width $\sim E_{C 0}$, since it costs an energy $\sim E_{C 0}$ to change the number of pairs at any dot. The exceptions are the discrete points at $N=(2 n+1)$, where a dot with charge $2 n e$ and $2(n+1) e$ has the same energy because the gate charge compensates the charges of extra Cooper pair in the dot. On this degeneracy point, a

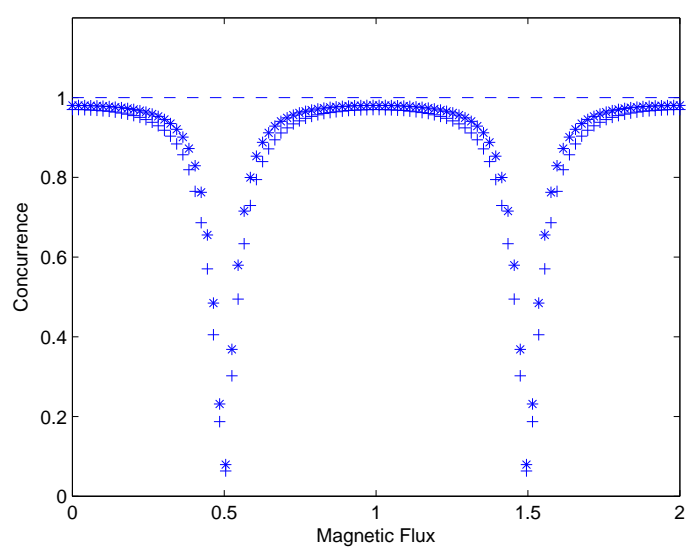

FIG. 1: Color online, concurrence vs magnetic flux at zero temperature for different values of $\delta V_{g}=0.0,0.2,0.5$ for the dashed bullet and + signs respectively. Here $E_{J}=1.0, E_{C 0}=$ 1.0 and $E_{Z 1}=0.4$.

small amount of Josephson coupling leads the system to the superconducting state.

Here we recast our basic Hamiltonians in the spin language, where each site of the dot is either empty or singly occupied. During this process we follow Ref. 25] and [29]. Now

$$
H_{J 1}=-2 E_{J 1}\left|\cos \left(\pi \frac{\Phi}{\Phi_{0}}\right)\right| \sum_{i}\left(S_{i}^{\dagger} S_{i+1}{ }^{-}+h . c\right),
$$

and

$$
\begin{gathered}
H_{E C 0}=\frac{E_{C 0}}{2} \sum_{i}\left(2 S_{i}{ }^{Z}-h\right)^{2} . \\
H_{E C 0}=-2 E_{C 0} \sum_{i} h_{i} S_{i}{ }^{Z} .
\end{gathered}
$$

Here $h_{i}=\frac{N_{i}-2 n-1}{2}$ allows the tuning of the system around the degeneracy point by means of gate voltage. We can tune the gate voltage in such a way that we can generate inhomogeneity in on-site charging energy. Without loss of generality we can also write the model Hamiltonian as

$$
H_{E C 0}=\sum_{i}\left(E_{C 0}+\delta V_{g}\right) S_{i}^{z}+\sum_{i}\left(E_{C 0}-\delta V_{g}\right) S_{i+1}^{z} .
$$

Where $\delta V_{g}$ is the variation of gate voltage around the lattice sites.

$$
H_{E C 1}=2 E_{Z 1} \sum_{i} S_{i}^{z} S_{i+1}^{z} .
$$

The total Hamiltonian of the system is

$$
\begin{aligned}
H= & 2 E_{J 1}\left|\cos \left(\pi \frac{\Phi}{\Phi_{0}}\right)\right| \sum_{i}\left(S_{i}^{\dagger} S_{i+1}^{-}+h . c\right)+2 E_{z 1} \sum_{i} S_{i}{ }^{z} S_{i+1}^{z} \\
& +\sum_{i}\left(E_{C 0}+\delta V_{g}\right) S_{i}{ }^{z}+\sum_{i}\left(E_{C 0}-\delta V_{g}\right) S_{i+1}^{z}
\end{aligned}
$$

Now we consider the Hamiltonian for $N=2$ case. We would like to write the Hamiltonian in the standard basis, 


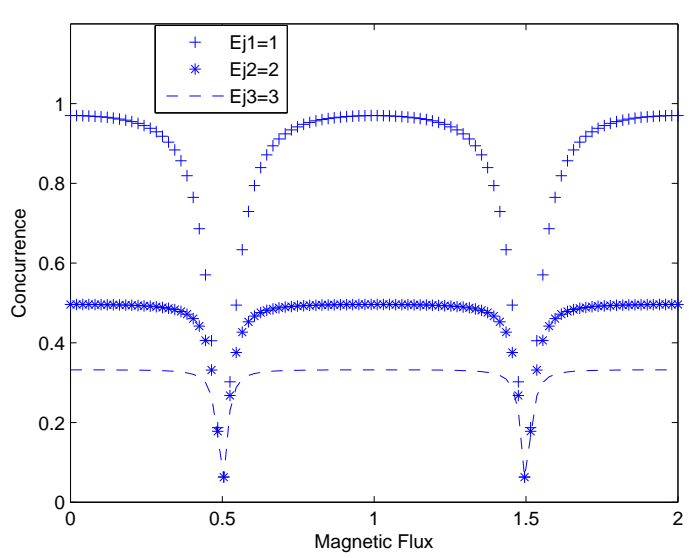

FIG. 2: Color online, concurrence vs. magnetic flux at zero temperature for different values of Josephson couplings. Here $E_{j}=1.0, \delta V_{g}=0.5$ and $E_{Z 1}=0.5$.

$|1,1>| 1,,0>,|0,1>| 0,,0>$

$H=\left(\begin{array}{cccc}E_{Z 1}+E_{C 0} & 0 & 0 & 0 \\ 0 & -E_{Z 1}+\delta V_{g} & B & 0 \\ 0 & B & -E_{Z 1}-\delta V_{g} & 0 \\ 0 & 0 & 0 & E_{Z 1}-E_{C 0}\end{array}\right)$

$B=2 E_{J 1}\left|\cos \left(\pi \frac{\Phi}{\Phi_{0}}\right)\right|$. The eigenstates of this two sites Hamiltonian are $\left.\left|\psi_{1}\right\rangle=|0,0>,| \psi_{1}\right\rangle=$ $|1,1>,| \psi_{3}>=\sqrt{\frac{1}{1+c_{1}^{2}}}\left(c_{1}|1,0>+| 0,1>\right), \mid \psi_{4}>=$ $\sqrt{\frac{1}{1+c_{2}^{2}}}\left(c_{2}|1,0>+| 0,1>\right)$. Where $c_{1}=\frac{\delta V_{g}-A}{B}$, $A=\sqrt{\delta V_{g}^{2}+4 E_{J}^{2}\left|\cos \left(\pi \frac{\Phi}{\Phi_{0}}\right)\right|^{2}}, c_{2}=\frac{\delta V_{g}+A}{B} . \quad E_{1}=$ $\frac{1}{2}\left(2 E_{Z 1}-2 E_{C 0}\right), E_{2}=\frac{1}{2}\left(2 E_{Z 1}+2 E_{C 0}\right), E_{3}=-E_{Z 1}-A$ $E_{4}=-E_{Z 1}+A$. If we consider the homogeneous system, i.e., there is no variation of gate voltage over the lattice sites. The two states $\left|\psi_{3}\right\rangle$ and $\left|\psi_{4}\right\rangle$ are the maximally entangled Bell states, i.e., $(1 / \sqrt{2})(|0,1>-| 1,0>)$ , $(1 / \sqrt{2})(|0,1>+| 1,0>)$. As we see from our analytical expression that ground state depends on the value of $E_{C 0}, E_{Z 1}$ and $A$. Ground state is in the disentangle state (product state) when the ground state energy is either $E_{1}$ or $E_{2}$, otherwise the system is in the entangle state. Thus for this superconducting quantum dot lattice system there is a transition between the disentangle state to entangle state due to the variation of the system parameters. We will see in due course of our study that the magnetic flux plays an important role in the transition between the disentangled state to the entangle state.

II. B Entanglement Study for Zero and Finite Temperature

Now we calculate the thermal entanglement of two arbitrary qubits $(N=2)$ of the superconducting quantum dot lattice. The density matrix of superconducting quantum dot lattice in equilibrium at temperature, $T$ is $\rho=\frac{1}{Z} \exp \left(-H / k_{B} T\right)$, where $H$ is the Hamiltonian of the system, $Z$ is the partion function of the system, $k_{B}$ is the Boltzmann constant. We calculate the concurrence to measure the entangle of two qubit system of superconducting quantum dot lattice following the reference of Wootters' formula $[14,15]$. The analytical expression for the concurrence is

$$
C=\max \left(0,2 \max \lambda_{i}-\sum_{i} \lambda_{i}\right)
$$

, where $\lambda_{i}$ is the square roots of the eigenvalues of the matrix $R=\rho\left(\sigma_{1}{ }^{y} \otimes \sigma_{2}{ }^{y}\right) \rho^{*}\left(\sigma_{1}{ }^{y} \otimes \sigma_{2}{ }^{y}\right)$. $\rho^{*}$ is the complex conjugate of $\rho$. The system is maximally entangled when $C=1$ and the system is in disentangle state when $C=0$. The density matrix of the system is

$$
\rho=\left(\begin{array}{cccc}
A_{1} & 0 & 0 & 0 \\
0 & B_{2} & C_{2} & 0 \\
0 & C_{2} & C_{3} & 0 \\
0 & 0 & 0 & D_{4}
\end{array}\right)
$$

The square roots of the eigenvalues of the matrix $\mathrm{R}$ are $\lambda_{1}=\lambda_{2}=\sqrt{A_{1} D_{4}}, \lambda_{3}=\sqrt{B_{2} C_{3}+C_{2}}$ and $\lambda_{4}=$ $\sqrt{B_{2} C_{3}-C_{2}}$. Where $A_{1}=\frac{1}{Z} e^{-E_{Z 1} / T}\left[\cosh \left(E_{C 0} / T\right)-\right.$ $\frac{1}{E_{C 0} T} \sinh \left(E_{C 0} / T\right) \quad D_{4}=\frac{1}{Z} e^{-E_{Z 1} / T}\left[\cosh \left(E_{C 0} / T\right)+\right.$ $\left.\frac{1}{E_{C 0} T} \sinh \left(E_{C 0} / T\right)\right] C_{2}=-\frac{B}{Z A} e^{\frac{E_{Z 1}}{T}} \sinh (A / T)$

$B_{2}=\frac{1}{Z} e^{E_{Z 1} / T}\left[\cosh (A / T)-\frac{\delta V_{g}}{A} \sinh (A / T)\right] ; \quad C_{3}=$ $\frac{1}{Z} e^{E_{Z 1} / T}\left[\cosh (A / T)+\frac{\delta V_{g}}{A} \sinh (A / T)\right]$. In this derivation we consider $k_{B}=1$.

Finally we obtain the formula for concurrence by using the relation Eq. 5,

$$
C=\frac{2}{Z} e^{E_{z 1} / T}\left(4 E_{J} \sinh \left(\frac{A}{T}\right)-e^{-2 E_{z 1} / T}\right)
$$

Now we calculate, the concurrence of the system by using the above relation and the final expression for the concurrence at $T=0$ is

$$
C=|B / A|
$$

Now we analyze the above equation of concurrence in different limit. If we consider the gate voltage in the lattice is homogeneous ,i.e., $\delta V_{g}=0$ that implies the concurrence $C(T=0)=1$. The system is always in maximally entangled state. For this situation magnetic flux has no effect to make a transition from entangled state to disentangled state.

If we consider the presence of inhomogeneous gate voltage, $\delta V_{g} \neq 0$ then the system has finite concurrence but it has not maximally entangled for any arbitrary magnetic flux. We observe an universality that at half-integer values of magnetic flux quantum, the concurrence is zero. The analytical expression which we derive for the concurrence is obtained satisfies the following relation

$$
{\sqrt{\delta V_{g}^{2}+4 E_{J}^{2}\left|\cos \left(\frac{\pi \phi}{\phi_{0}}\right)\right|}}^{2} \geq E_{C 0}-2 E_{Z 1}
$$




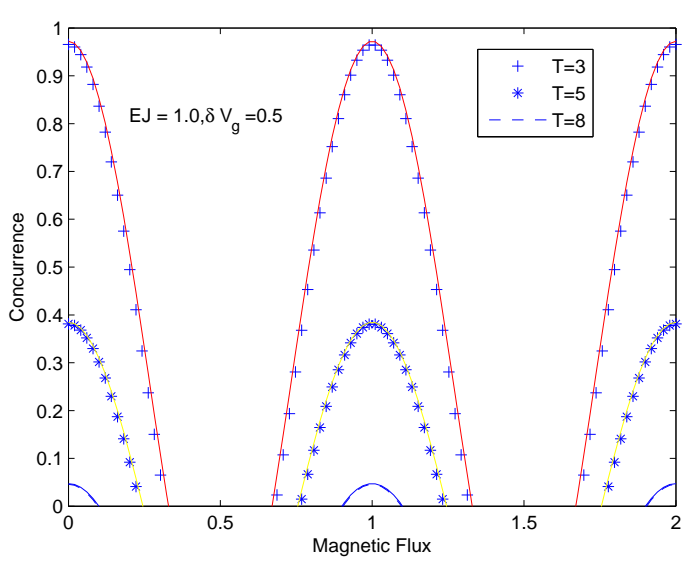

FIG. 3: Color online, concurrence vs. magnetic flux at different finite temperatures. Here $E_{J}=1.0, \delta V_{g}=0.5, E_{C 0}=1.0$ and $E_{z 1}=0.5$.

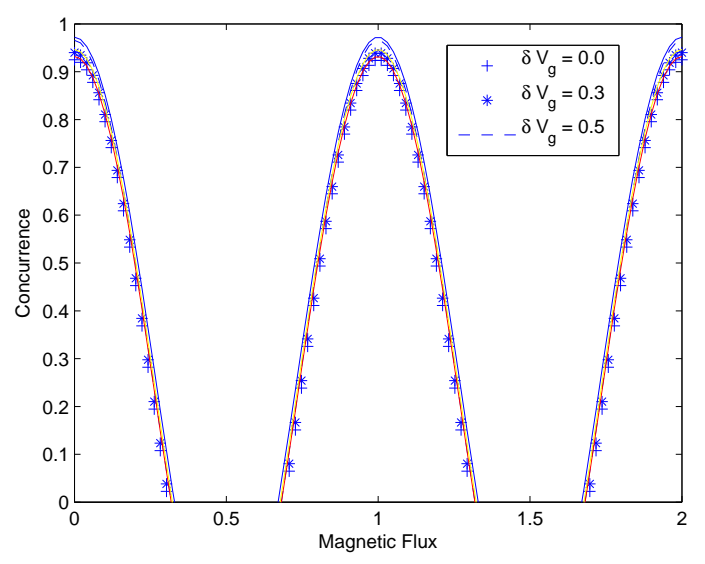

FIG. 4: Color online, concurrence vs. magnetic flux for different inhomogeneity of gate voltage in SQD lattice. Here $E_{J}=1.0, E_{C 0}=1.0$ and $E_{z 1}=0.5$.

From the analysis of the above equation we get the constraint of magnetic flux that to obey the concurrence relation system.

$\phi \geq \frac{\phi_{0}}{\pi} \cos ^{-1} \frac{\sqrt{\left(E_{C 0}-2 E_{Z 1}-\delta V_{g}\right)} \sqrt{\left(E_{C 0}-2 E_{Z 1}+\delta V_{g}\right)}}{2 E_{J}}$

Now we present our results and physical explanations. Fig. 1, shows the variation of concurrence with magnetic flux for different values of $\delta V_{g}$. We observe from the figure that the system is maximally entangled Bell state when there is no inhomoginity in the applied voltage in the lattice. This maximally entangled Bell state is independent of the applied magnetic flux. We also observe from this figure that a finite inhomogeneity in the applied gate voltage introduces a transition from entangled state to disentangled state. In the entanglement phase system is almost maximally entangled state.
Fig. 2 shows the variation of concurrence with magnetic flux for different values of Josephson couplings. We observe from our study that concurrence is large for the smaller values of ratio between the Josephson coupling to on-site Coulomb charging energies. As we have obtained from our previous studies that this large ratio of $\frac{E_{J}}{E_{C 0}}$ favors the Luttinger liquid and superconducting phase of the system and the smaller one favors the Mott insulating density wave phases of the system $[25,26]$. Thus it is clear from our study that entanglement of the system is large for Mott-insulating phase of the system where the Cooper pairs are localized. But the collapse of entanglement at the half-integer magnetic flux quantum has the universal feature for all values of Josephson couplings.

Fig. 3 shows the variation of concurrence with magnetic flux at different temperatures. It reveals from our study that the concurrence is smaller for the higher values of temperature and at the same times the system is in the disentangled state for wider region of magnetic flux. The region of disentanglement state is larger for the higher values of temperature. The solid lines in the figures are for the considerations of superconducting Coulomb blocked effect induced co-tunneling effect in the system. We observe that co-tunneling effect has not drastic effect to change entangle transition of the system at the quantitative level. For ground state entanglement, the effect of co-tunneling effect is not explicit in the analytical expression (Eq. 8).

Fig. 4 shows the variation of concurrence with magnetic flux for different values of $\delta V_{g}$. Here we observe that the presence and absence of gate voltage inhomogeneity has no appreciable for the transition from entangle state to disentangle state. This behavior at finite temperature is in contrast with the behavior at zero temperature where the system is always entangled state for homogeneous gate voltage.

\section{SUMMARY AND CONCLUSIONS}

We have studied the entanglement of a two qubit system of a superconducting quantum dot lattice. We have observed the magnetic flux dependent entangled state to disentangled state transition at zero and finite temperature. We find an important constraint on magnetic flux during the study of ground state entanglement. We have observed that at zero temperature that the system is always maximally entangled state when there is no-inhomogeneity in the gate voltage in the lattice sites whereas the presence and absence of inhomoginity has no effect at finite temperature. We have found out the universal feature for half-integer magnetic flux quantum.

The author would like to acknowledge the CCMT of the physics department of IISc for extended facility use. The author would like to acknowledge Dr. R. Srikanth for reading the manuscript critically. 
[1] L. Amico, R. Fazio, A. Osterloh and V. Vedral, Rev. Mod. Phys 80, 517 (2008).

[2] M. Lewenstein et al., Adv. Phys 56, 2 (2007).

[3] R. Fazio and H. van der Zant, Phys. Rep 355, 235 (2001).

[4] I. Bose and A. Tribedi, Lect. Notes Phys 802, 177-200 (Springer-Verlag, Heidelberg 2010).

[5] S. J. Gu, arXiv:0811.3127.

[6] H. T. Quan et al., Phys. Rev. Lett 96, 140604 (2006);

[7] P. Zanardi and N. Paunkovic, Phys. Rev. E 74, 031123 (2006).

[8] M. Cozzini, R. Ionicioiu and P. Zanardi, Phys. Rev. B 76, 104420 (2007).

[9] H. Q. Zhou, e-print arXiv:0704.2945.

[10] S. Chen, L. wang, S. J. Gu and Y. Wang, Phys. Rev. E 76, 061108 (2007).

[11] M. A. Nielsen, Phys. Rev. A 63, 022114 (2001).

[12] X. Wang, Phys. Rev. A 64, 012313 (2001).

[13] G. L. Kamta and A. F. Starace, Phys. Rev. Lett 88, 107901 (2002).

[14] K. M. O'Connor and W. K. Wootters, Phys. Rev. A 63, 052302 (2001).

[15] W. K. Wootters, Phys. Rev. Lett 80, 2245 (1998).

[16] Y. Sun, Y. Chen and H. Chen, Phys. Rev. A 68, 044301 (2003).

[17] Y. Yeo, Phys. Rev. A 66, 062312 (2002).

[18] D. V. Khveshchenko, Phys. Rev. B 68, 193307 (2003).
[19] G. Zhang, S. Li and J. Liang, Opt. Commun. 245, 457 (2005).

[20] S. Bose, Phys. Rev. Lett 91, 207901 (2003).

[21] M. Christandl. N. Datta, A. Ekert and A. J. Landahl, Phys. Rev. Lett 92.

[22] D. Loss and D. P. DiVincenzo, Phys. Rev. A 57, 120 (1998).

[23] Guo-Feng Zhang and Shu-Shen Li, Phys. Rev. A 72, 034302 (2005).

[24] G. H. Yang, W. B. Gao, L. Zhou, H. S. Song, arXiv:quant-ph/0602051.

[25] S. Sarkar, Phys. Rev. B 75, 014528 (2007).

[26] S. Sarkar Eur. Phys. J. B, 67, 559 (2009); arxiv0805.4713).

[27] S. Sarkar, Renormalization Group study of mesoscopic SQUID array in Quantum Phase Transition and Dynamics: Quenching, Anneling and Quantum Computation, Springer, 2010.

[28] E. Chow et al., Phys. Rev. Lett 81, 204 (1998).

[29] L. I. Glazman and A. I. Larkin, Phys. Rev. Lett. 79, 3786 (1997).

[30] H. Jaeger, D. Haviland, B. Orr, A. Goldman, Phys. Rev. B 40 (1989), 182.

[31] B. G. Orr, H. M. Jaeger, A. M. Goldman and C. G. Kuper, Phys. Rev. Lett 56, (1986) 378. 